\title{
Telescope Guiding with a HyViSI H2RG Used in Guide Mode
}

\author{
Lance M. Simms ${ }^{a}$, Donald F. Figer ${ }^{b}$, Brandon J. Hanold ${ }^{b}$, Steven M. Kahn ${ }^{a}$, D. Kirk \\ Gilmore $^{a}$ \\ ${ }^{a}$ Stanford Linear Accelerator Center, Menlo Park, CA, 94025 USA; \\ ${ }^{b}$ Rochester Imaging Detector Laboratory, Chester F. Carlson Center for Imaging Science, RIT, \\ Rochester, NY 14623 USA
}

\begin{abstract}
We report on long exposure results obtained with a Teledyne HyViSI H2RG detector operating in guide mode. The sensor simultaneously obtained nearly seeing-limited science data while also guiding the Kitt Peak $2.1 \mathrm{~m}$ telescope. Results from unguided and guided operation are presented and used to place lower limits on flux/fluence values for accurate centroid measurements. We also report on significant noise reduction obtained in recent laboratory measurements that should further improve guiding capability with higher magnitude stars.
\end{abstract}

Keywords: HyViSI, H2RG, SIDECAR ASIC, Guide Mode

\section{INTRODUCTION}

\subsection{H2RG Hybrid Visible Silicon CMOS Detector}

The Teledyne Imaging Sensors (TIS) Hybrid Visible Silicon Imager (HyViSI) H2RG and H4RG detectors have been well discussed in the literature. For an introduction to these sensors, the reader is referred to Bai et al. (2003), ${ }^{1}$ and to Bai et al. $(2008)^{2}$ for the latest in HyViSI technology. Simms et al. ${ }^{3}$ and Dorland et al. ${ }^{4}$ characterized the first generation 10 micron pixel $4096 \times 4096 \mathrm{HyViSI}$ H4RGs in the context of astronomy and found they suffered from very high leakage currents. TIS has since released a second generation, which is expected to reduce this problem by an order of magnitude (see Dorland et al..$^{5}$ ). The 18 micron pixel $2048 \times$ 2048 H2RG does not suffer from high leakage currents and was shown to have performance levels suitable for astronomy by Dorn et al. in $2008 .^{6}$

Because the H2RG HyViSI has proven itself ready for visible and near infrared astronomy - and because it possesses CMOS windowing capability that allows for fast readout of a star independent of location on the detector - it has been chosen as the baseline sensor for the Large Synoptic Survey Telescope (LSST) guider subsystem. In order to test the H2RG in this role, the engineering grade detector H2RG-32-147 was obtained from TIS and tested extensively in the Rochester Imaging Detector Laboratory (RIDL) and at the Kitt Peak $2.1 \mathrm{~m}$ telescope. The properties of H2RG-32-147, which were obtained with the same methodology outlined in Simms et al., ${ }^{3}$ are listed in Table 1. Unless otherwise noted, all measurements were taken with a backside contact voltage of $V_{S U B}=15 \mathrm{~V}$ at 170 Kelvin in Full Frame mode. For some of the astronomical observations, the detector was tested in its guide mode to guide the telescope, which is the subject of the next section.

Table 1. Standard detector metrics for H2RG-32-147 at $170 \mathrm{~K}$. These values were measured after the improvements mentioned in Section 4.2. The inter-pixel capacitance (IPC) values represent the ratio of signal measured in the specified neighbors, $I_{\text {neighbor }}$, to the signal measured in the center pixel, $I_{\text {center }}$. They were obtained by analyzing single pixel Fe ${ }^{55}$ events. Quantum Efficiency (QE) measurements are taken from Dorn et al. ${ }^{6}$ for another HyViSI H2RG.

\begin{tabular}{||l|c||l|c||}
\hline CDS Read Noise & $10 e^{-} \mathrm{rms}$ & Fowler 5 Read Noise & $5 e^{-}$rms \\
Dark Current & $0.20 e^{-} / \mathrm{sec} /$ pix & Linearity & $1 \%$ over $85 \%$ of full well \\
Pixel Responsivity & $10.5 \mu V / e^{-}$ & Well Depth & $85,000-105,000 e^{-}$ \\
IPC (Nearest Neighbors) & 0.08 & IPC (Diagonal Neighbors) & 0.02 \\
Latent Image Max. Duration & 340 seconds & QE $(400 / 700 / 1000 \mathrm{~nm})$ & $59 \% / 95 \% / 19 \%$ \\
\hline
\end{tabular}

Work supported in part by US Department of Energy contract DE-AC02-76SF00515. 


\subsection{Telescope Guiding and the H2RG Guide Mode}

Modern telescopes use very precise mechanical motors to track celestial objects across the sky while their location changes due to the rotation of the Earth*. The job is slightly easier for equatorial-mount telescopes that only need to adjust in right ascension (RA) than it is for altitude-azimuth telescopes that must adjust in right ascension and declination (DEC) to compensate for the sidereal motion of the heavens. However, even for equatorial-mount telescopes with the most sophisticated motors available it is an imperfect process. The majority of telescopes must keep their pointing accurate to less than an arcsecond in order to prevent "smearing" of stars and galaxies while they are being imaged.

The widespread solution in astronomy is to use a "guide star" to correct for any errors in the telescope tracking, the idea being that if the sidereal motion is being properly accounted for, a given star should stay in the same physical location $\left(x_{o}, y_{o}\right)$ on the focal plane of the telescope. If any movement in the location $(\Delta x, \Delta y)$ is detected (by a CCD or other imaging detector), tip/tilt corrections are made in the pointing of the telescope in order to bring the star back to its original location and make $\Delta x=\Delta y=0$. Usually a sufficiently bright guide star is used to track offsets while a very dim object is being imaged in a long exposure.

If a CCD is being used as a "science sensor" to make the long duration exposure, it cannot be simultaneously used to guide. Accessing the pixels that contain the guide star would result in a destructive read of the entire array. Thus, a separate "guide sensor" is required to measure $\Delta x$ and $\Delta y$. In contrast, because of the nondestructive readout and random access capabilities inherent in CMOS detector architecture, a CMOS device can act as both the guide and science sensor simultaneously. In particular, the Teledyne HxRG multiplexer has been designed with a special "guide mode" to accomplish this. ${ }^{7,8}$ Such capability is of great advantage for large focal plane arrays that consist of many detectors since any one of them can be used to track a guide star while it simultaneously participates in the science exposure.

Another benefit of the guide mode operation is that saturated pixels can be reset while the rest of the array integrates light. This prevents the dark current from hot pixels and photocharges generated by bright stars from blooming into neighboring pixels and prevents output crosstalk. This can be very advantageous for long exposures of regions where both bright stars and very dim objects are present.

\subsection{Purpose of Experiment}

Laboratory measurements and calculations can provide some indication of the impact that IPC, read noise, nonlinearity, and image persistence will have on the ability of the H2RG to guide a telescope. For instance, convolving the pixel impulse response with a simulation of the telescope point spread function and folding in read and shot noise will provide an estimate of the expected signal to noise and centroid accuracy for a stable point source. However, it is not immediately clear what subtle effects atmospheric turbulence and scintillation will induce when latent images are present. For instance, if atmospheric effects displace the stellar image by a few pixels for several seconds, the latent image that forms might cause errant centroid values for subsequent measurements after the star has returned to its original location on the detector. Reproducing such effects would require very elaborate simulations or a complicated laboratory setup. And in fact, analytical models and simulations used to fit latent images based upon the flux and fluence of the offending image result in large errors, so this might not be a valid option to pursue.

To directly measure the impact of these second order effects and see whether or not the HyViSI H2RG can accurately track a star, we used H2RG-32-147 to guide the Kitt Peak 2.1m telescope. Operating in guide mode, it was able to simultaneously obtain high dynamic range exposures of the full field and track a guide star over long periods of time. In the following sections, we describe the method of implementation and show measurements made from long exposures with and without guiding. We also show that our guide mode measurements were made with sub-optimal operating conditions and predict that significant improvement in guiding on faint stars is expected based on recent results.

\footnotetext{
${ }^{*}$ This motion is referred to as sidereal motion.
} 


\section{EXPERIMENT AND CALIBRATION}

\subsection{Experimental Setup}

At the Kitt Peak 2.1m telescope in Tucson, AZ. in November of 2007 we observed with a H1RG HyViSI detector (H1RG-022) and in December of 2007 with a H2RG HyViSI detector (H2RG-32-147). In both cases we removed the standard instrument rack from the telescope and mounted our dewar to it. In addition to the detector, the dewar housed a filter wheel with $i, g$, and $y$ band filters, a blank plate for dark acquisition, and an $\mathrm{Fe}^{55}$ source for calibration (see Simms et al. ${ }^{3}$ for filter specifications). For both runs we placed the detector at the Cassegrain focus. With $18 \mu \mathrm{m}$ pixels for both the H1RG and H2RG, the plate scale was $0.232^{\prime \prime}$.

To attach the dewar to the Kitt Peak $2.1 \mathrm{~m}$ telescope, it was necessary to remove the CCD guider from the instrument rack. As a consequence, the default configuration for our observing runs had the Telescope Control System (TCS) tracking the sky at the sidereal rate with no guiding offsets being issued to account for errors in tracking. Many of our exposures were taken in this mode. Only during our last observing run with the H2RG did we implement guide mode in order to track a guide star.

\subsection{Telescope Calibration with Multiple Windows}

High speed photometric measurements of the Delta Scuti star BE Lyn (V 8.8) were made during the observing run with H2RG-32-147. On the night of December 17, 2007, BE Lyn and the nearby reference star GSC 0342500544 (V 11.1) were simultaneously imaged at a rate of $4 \mathrm{~Hz}$ over the course of several hours. The two stars are separated by approximately $2^{\prime}$. A separate $61 \times 61$ guide window was used for each star, in a sequence where the first and second windows were reset in series, read once in series, and read once again in series to provide a Correlated Double Sample (CDS). This sequence was executed repeatedly and was only interrupted when it was necessary to write the data to a FITS file. In addition to providing temporal flux measurements, from which the period of BE Lyn was measured in $i$ band, this data inadvertently provided a detailed look at the telescope tracking.

The star centroids calculated from this data show that there are significant tracking errors for both right ascension and declination. In fact, the errors were so large that it was necessary to manually offset the telescope while the observations were being made. This was possible since the data was being displayed in real time on a computer monitor. The errors are quantified as drifts between the telescope tracking rate and the sidereal rate. For periods of about 20 minutes or less, the drift in DEC can be fit with a straight line; any longer than this and a curvature becomes evident, especially as the telescope crosses the meridian. The drift in RA has both a long period linear component and a short period sinusoidal one. The drifts $\Delta_{D E C}$ and $\Delta_{R A}$, measured in arcseconds, can be reasonably well fit over timespans less than 20 minutes with the functions:

$$
\begin{aligned}
\Delta_{D E C} & =m_{D E C} * t \\
\Delta_{R A} & =m_{R A} * t+A \sin \left(2 \pi f_{o s c} t\right),
\end{aligned}
$$

where $m_{D E C}$ and $m_{R A}$ are the linear slopes of the drifts, and $A$ and $f_{o s c}$ are the amplitude and frequency of the short period oscillation in RA, respectively.

For 12 piecewise fits over the 4 hour time span, the average slopes were found using least square fits to the data. The magnitude of $m_{R A}$ varied between $0.110^{\prime \prime} /$ minute and $0.950^{\prime \prime} /$ minute, and was greatest right before crossing the meridian. The sign of $m_{R A}$ was always negative, indicating that the telescope was lagging the movement of the sky. The sign of $m_{D E C}$ changed several times over the 4 hour period. Its magnitude ranged from 0.028 "/minute to 0.39 "/minute, and was also largest while the telescope crossed the meridian.

After removing the linear component in the RA data, a discrete Fourier transform of its autocorrelation was performed for each piecewise fit. The frequency was determined to be $f_{\text {osc }}=0.00833 \mathrm{~Hz}$, corresponding to a period of 120 seconds. This frequency was extremely consistent throughout the data and so was the value of the amplitude, $A=.5104^{\prime \prime}$. Although the residuals in the fit show that the oscillations are not purely sinusoidal (more time is spent in the leading portion of the curve than the lagging portion), the amplitude and frequency provide an estimate on the reduction in image quality. A fit to the data for a 16 minute period is shown in Figure 1. It should also be noted that autorcorrelations in the DEC drift show power at $f_{\text {osc }}$, indicating that the two coordinate motions are coupled. However, the amplitude is much lower, at about the level of the high frequency components induced by atmospheric effects. 

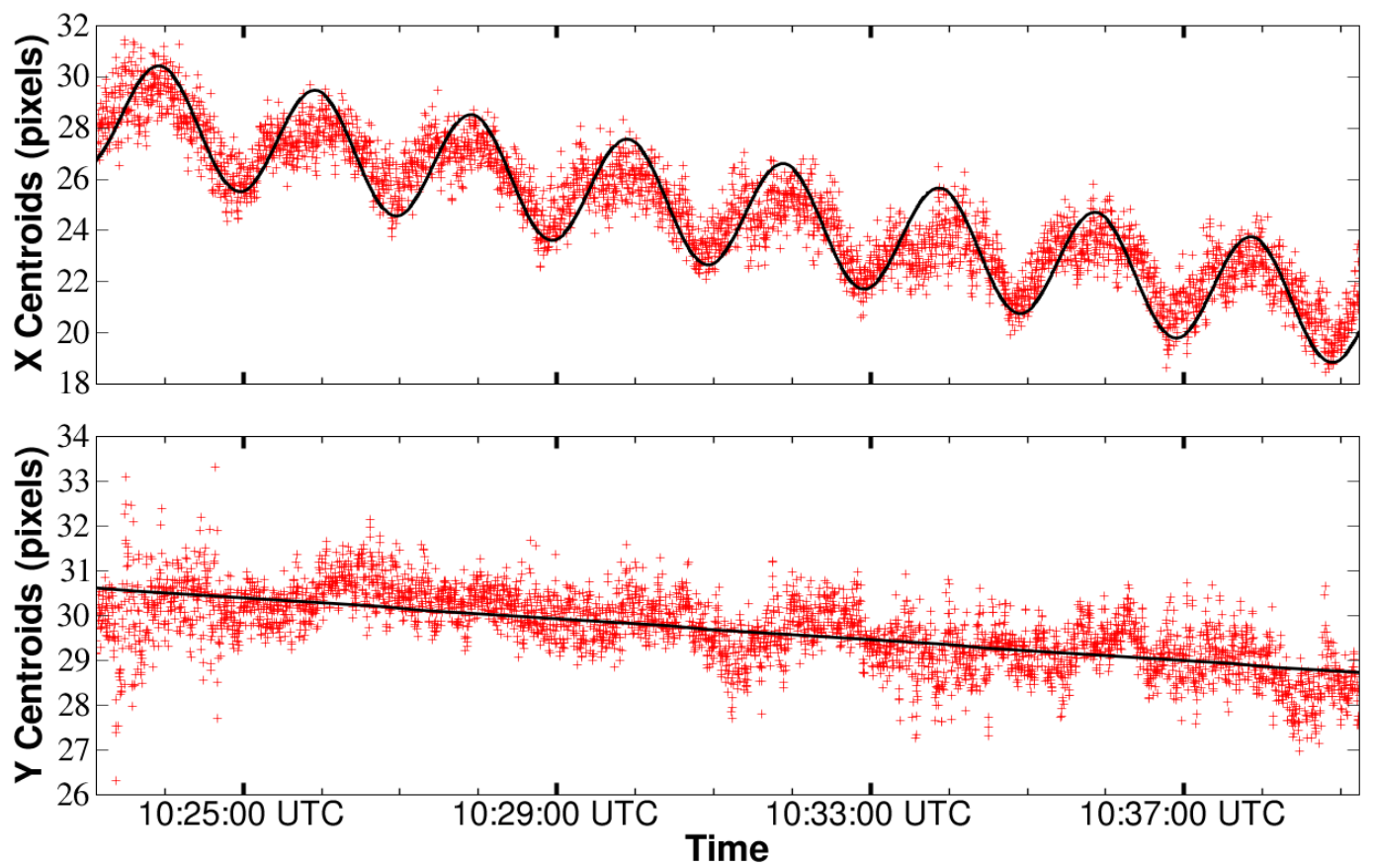

Figure 1. The telescope drifted significantly over a 20 minute period and showed sizable oscillations without the assistance of a guider. The centroids from images taken at $4 \mathrm{~Hz}$ are shown as plus marks. The black lines show the fits to the drifts from Eq. 1 with $m_{R A}=-0.108^{\prime \prime} /$ minute and $m_{D E C}=-0.026^{\prime \prime} /$ minute.

\subsection{Expected Point Spread Function}

The tracking error functions in Eq. 1 can be used directly to predict the image quality for unguided and guided exposures. The full data set collected during the observing runs with H1RG-022 and H2RG-2-147 shows that the system point spread function (PSF) is Gaussian and seeing-limited for intermediate length exposures (long enough to average out atmospheric turbulence and short enough to avoid degradation from tracking error). For long exposures, the center of the gaussian profile is assumed to drift at the rates $m_{D E C}$ and $m_{R A}$ in DEC and $\mathrm{RA}$, respectively, and oscillate in RA at a frequency of $f_{o s c}$. For an exposure of length $t_{\text {exp }}$, the resulting point spread function will thus be:

$$
I(x, y)=\int_{0}^{t_{e x p}} I_{o} \exp \left(\frac{-\left(x-m_{R A} * t-A \sin \left(2 \pi f_{o s c} t\right)\right)^{2}}{2(\epsilon / 2.35)^{2}}+\frac{-\left(y-m_{D E C} * t\right)^{2}}{2(\epsilon / 2.35)^{2}}\right) d t,
$$

where $\epsilon$ is the Full Width at Half Max (FWHM) due to the average seeing in arcseconds, and $I_{o}$ is the peak intensity of the stellar image. Numerical solutions to this integral were obtained for representative values of $\epsilon$, $t_{\text {exp }}, m_{R A}, m_{D E C}$ in order to predict the image quality in both guided and unguided operation.

To quantitatively describe the PSF for each solution, a $21 \times 21$ box around the centroid of the intensity distrubution is used to compute the second moments $I_{x x}, I_{y y}$, and $I_{x y}$, where:

$$
I_{i j}=\frac{\sum_{n, m} x_{i}(n, m) x_{j}(n, m) I(n, m)}{\sum_{n, m} I(n, m)} .
$$

From these, the ellipticity vector is calculated from its components, $e_{1}$ and $e_{2}$, according to the prescription in Bacon et al..$^{9}$ :

$$
e_{1}=\frac{I_{x x}-I_{y y}}{I_{x x}+I_{y y}}, \quad e_{2}=\frac{I_{x y}}{I_{x x}+I_{y y}}
$$


The magnitude of ellipticity, $e$, and its angle, $\theta$, are given by

$$
e=\left(e_{1}^{2}+e_{2}^{2}\right)^{1 / 2}, \quad \tan 2 \theta=e_{2} / e_{1}
$$

In addition to these measurements, the radial profiles around the centroid are fit with one-dimensional Gaussian and Moffat functions. The FWHM from the fits, along with the second moments, measure the overall extent of the PSF, while the ellipticity measures the asymmetry in RA and DEC. Together these quantities adequately describe the PSF.

\subsubsection{Unguided Operation}

It is fairly straightforward to see from equation 1 that if the telescope is not guided, the tracking errors will quickly produce a PSF that is broadened beyond the seeing disc and elongated along the direction of the drift. However, it is not trivial to quantitatively describe the effect the drift will have on its shape. For two representative drift rates observed during the photometric measurements of VBELyn, the ellipticity and FWHM obtained from a Gaussian fit were calculated for a range of atmospheric seeing values, $\epsilon$, assuming $t_{e x p}=1800 \mathrm{~s}$. The results are shown in the top two curves of Figure 2.

The plots show that the ellipticity can easily exceed 0.2 when the blurring due to seeing is not large enough to mask the sinusoidal oscillation and drift of the telescope. This effect is more pronounced when $m_{R A}>m_{D E C}$ since the centroid motion along the $\mathrm{x}$ axis of the detector is far greater than the motion along the $\mathrm{y}$ axis during the exposure. It should also be noted that at a given value of $\epsilon$ the ellipticity will be an increasing function of $t_{\text {exp }}$, as the smearing along the direction of drift increases, while in the transverse direction it remains constant. For short exposures $\left(t_{\exp }<1 \mathrm{~m}\right)$, a Gaussian shape is preserved for typical seeing values.
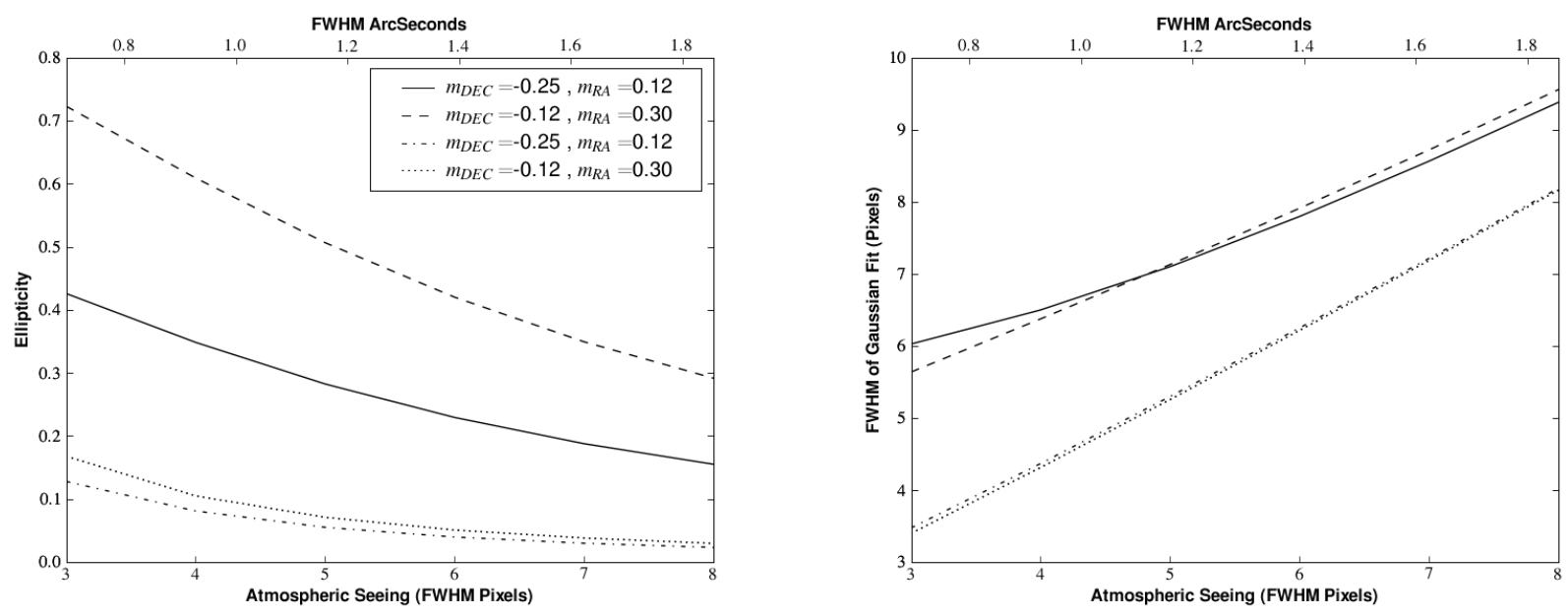

Figure 2. Expected ellipticity (left) and FWHM (right) derived from a Gaussian fit with a drifting center for a 30 minute exposure. The drift rates $m_{D E C}$ and $m_{R A}$, expressed in "/minute are shown in the legend. The top two curves in each plot represent the results for the cases where the telescope is allowed to drift. The bottom two show the calculation results for the cases where the drift is corrected (by recentering the Gaussian) whenever the pointing has exceeded $1.3^{\prime \prime}$ in $R A$ or $D E C$.

\subsubsection{Guided Operation}

During our last observing run we implemented guide mode with H2RG-32-147 and issued offsets to the telescope to correct for tracking error. In our configuration, one set of IDL scripts was used to collect data from the SIDECAR ASIC through its USB interface and another set of IDL scripts communicated to the TCS via a RS-232 serial port connection. Centroids were calculated in IDL as the detector was integrating up the ramp and an offset was issued to the telescope if it appeared that the telescope was drifting. 
The TCS for the $2.1 \mathrm{~m}$ accepts offsets as small as .1 arcseconds. However, due to telescope motor hysteresis and other mechanical effects, the minimum offset which can be issued with precision is not this small. While a precise value is not known, it is definitely less than 1 arcsecond. After some initial measurements, we found that the best results were obtained when we issued offsets only if the centroid of the star had moved by $0.3^{\prime \prime}$, which corresponds to about 1.3 pixels with the $0.232^{\prime \prime}$ plate scale. On average we found that an offset in RA was needed every 20-50 seconds. The declination adjustment varied between once per 1-5 minutes depending on the altitude of the pointing.

To predict the quality of the PSF with the adjustments described, Equation 1 is not used directly. Instead, it is broken up into a total of $t_{\text {exp }} / t_{\min }$ adj. separate integrals, where $t_{\min }$ adj. $=20 \mathrm{~s}$ is the interval of time between the most frequent adjustments in $R A$. For each integration, the center in $\mathrm{x}$ of the Gaussian is set back to zero.

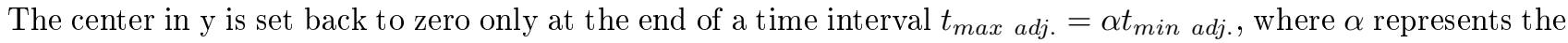
ratio of $R A / D E C$ adjustment frequencies. This number ranged between 3 and 15 depending on the pointing. The phase of the sinusoidal motion is preserved between successive integrations to best represent the observed behavior of the telescope motion after small adjustments.

The results of calculations for which $t_{m i n_{a} d j .}=22 \mathrm{~s}, \alpha=12$, and $t_{\exp }=1800 \mathrm{~s}$ are shown in the bottom two curves of Figure 2. The linear drift rates are the same as the two cases represented in the unguided calculation. The ellipticity and FWHM are greatly diminished as expected. However, the assymmetric centroid motion along the $\mathrm{x}$ and $\mathrm{y}$ axes yields a nonzero ellipticity. For small linear drift rates, the sinusoidal motion along the $\mathrm{x}$-axis is actually the greatest contributor. This can be understood by considering the times immediately after the telescope has been corrected in $R A$. Since the phase of the oscillatory motion is preserved after adjustment, the pointing of the telescope following a correction will in some cases swing in the opposite direction of the linear motion and contribute to a greater blurring in the $\mathrm{x}$ direction.

\section{RESULTS}

\subsection{Results without Guide Mode}

Several long exposures were taken without any guiding. The slopefit ${ }^{\dagger}$ from such an exposure, taken in $i$ band, is shown on the left in Figure 3. No dark subtraction or flat field has been applied so hot pixels, defects, and nonuniformity are present in the image. As exemplified by this figure, tracking errors clearly show up in long exposures taken with H1RG-022 and H2RG-32-147. Because the telescope leads or lags the sidereal motion, the light from the stars is smeared out over the pixels so that they have a "jelly-bean" like appearance. We will provide a quantitative description of this in Section 3.3.

The bright star in this exposure is SAO 54817, a star with magnitude 6.55 in the V-band and 4.717 in the J-band. The flux falling on the detector was thus somewhere between $400-900 \mathrm{~mW} / \mathrm{m}^{2}$ in $i$ band. By the end of the exposure, the light from this star has saturated a circular region out to a radius of 50 pixels. For the dimmer stars, image blur due to the tracking error is clearly evident, as evidenced in the top right image of Figure 3.

\subsection{Results with Guide Mode}

An example guide exposure is shown in Figure 4. It is a slopefit (no dark subtracted or flat field to remove hot pixels and defects) to a $2597 \mathrm{~s} i$ band exposure of an $8^{\prime} \mathrm{x} 8^{\prime}$ field centered around the star IRAS $09595+2513$, which was used as the guide star. The magnitude in $\mathrm{V}$ of this star was not found in any catalogs, but is listed as 5.807 in $\mathrm{J}$ and 4.594 in $\mathrm{K}$. It has colors of a late $\mathrm{M}$ star, so $\mathrm{V}-\mathrm{K} \sim 6.2$, and thus $\mathrm{V} \sim 10.8 .^{10}$ Based upon these colors, in $i$ band we expect a flux of $20-170 \mathrm{~mW} / \mathrm{m}^{2}$ was falling on the detector.

For this exposure, 220 reads of the full frame were recorded and in between them 200 CDS frames of the window were read out. The window was $35 \times 37$ pixels and the time for a CDS pair was $t_{s w}=40.9 \mathrm{~ms}$, which corresponds to approximately $25 \mathrm{~Hz}$ sampling. Using the flux estimate above, at this sampling rate the maximum fluence was $0.8-6.8 \mathrm{~mJ} / \mathrm{m}^{2}$. All of the 200 frames were coadded and the centroid of the star was calculated from the final sum using a $9 \times 9$ box at the middle of the full window, which yielded an effective guiding rate of 0.12

\footnotetext{
${ }^{\dagger}$ A slopefit is a two-dimensional image formed by fitting a slope to the ramp of each pixel in a three-dimensional datacube. See Simms et al. for details on the computation used. ${ }^{3}$
} 
Hz. This is slow in comparison to typical guiding rates; atmospheric disturbances average out on the order of seconds and any centroid displacement after that length of time is attributed to tracking error. However, we did not issue offsets faster than this because offsets less than $0.3^{\prime \prime}$ were found to be inaccurate, resulting in increased blurring of the image (see Section 2.3.2).

\subsection{Comparison between Exposures with and without Guide Mode}

Figure 5 shows a close up-image of a star taken from each of the long exposures along with a quantitative comparison of the stars in terms of their ellipticity and FWHM. We consider these here along with the difference in limiting magnitude in each exposure and the saturation of pixels surrounding the central star.

Ellipticity The stars in the guide mode slopefit do have a slight average ellipticity of 0.06 along the $\mathrm{x}$ direction. We note that this is the direction that coincides with right ascension: the direction that was corrected more frequently. However, the ellipticity is 3 times times smaller than it was when the telescope was not being guided. In the latter case the ellipticity of the stars was clearly induced by tracking errors, particularly the oscillations discussed in section 2.3. It is clear that the guiding succeeded in diminishing the ellipticity of the stars.

Full Width at Half Maximum As shown in Figure 5 the FWHM is significantly smaller when the telescope was guided. It is just slightly larger than the seeing of $1.0^{\prime \prime}(4.3$ pixels) recorded for the night. The seeing on the night when we imaged SA0 54817 was actually slightly better at approximately 0.9 ". We thus conclude that the smaller image blur is a result of guiding rather than atmospheric conditions.

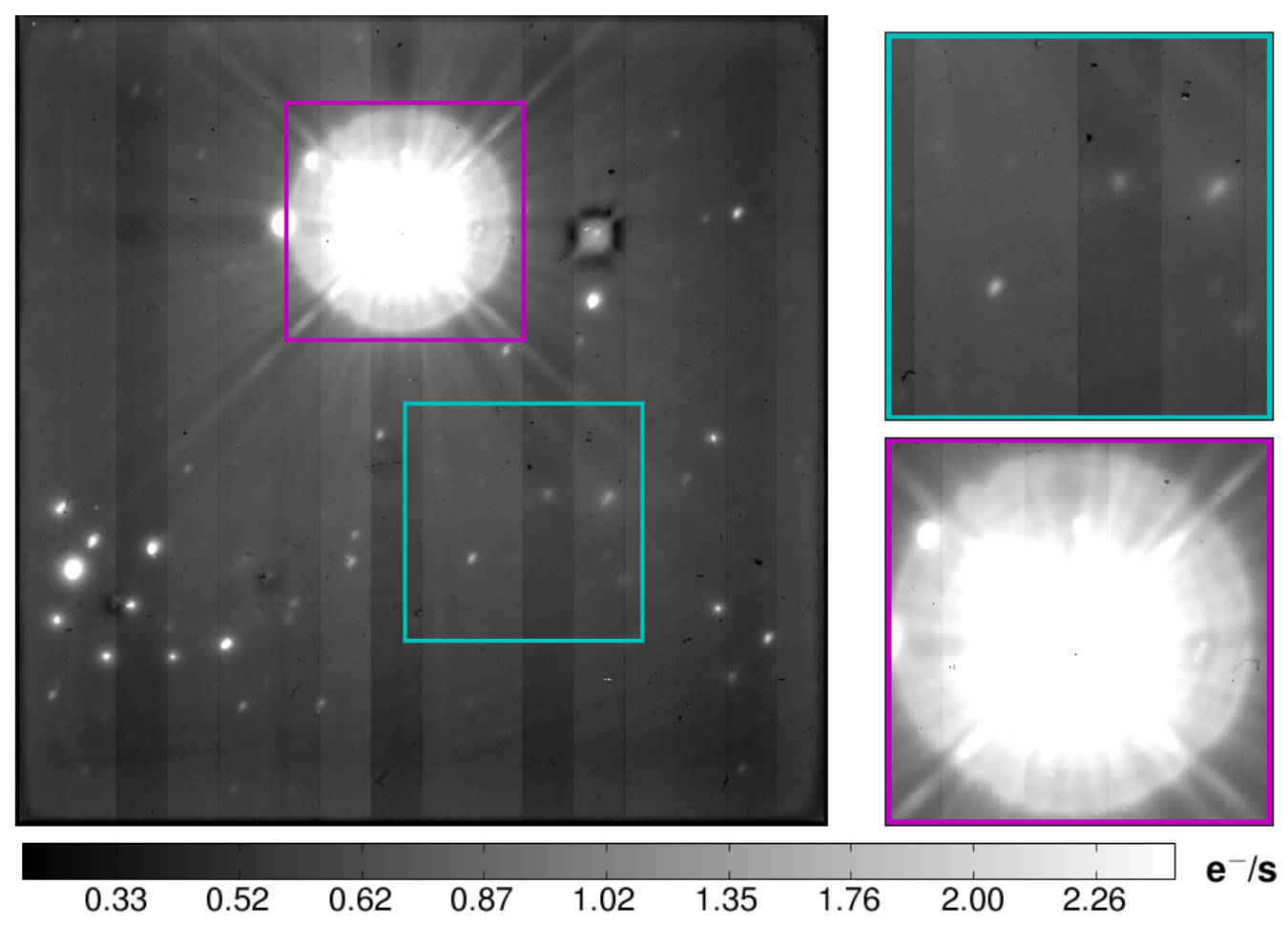

Figure 3. (Left) Full frame slopefit to a 1770 s exposure taken with H1RG-022 with no guiding. (Right) A zoomed view of the box enclosing the very saturated star SAO 54817 is shown at bottom right. The light from this star has consumed a circular region of pixels with a radius of 50 pixels. The box at upper right shows an area away from the guide window that contains three stars of irregular shape. Both boxes are $300 \times 300$ pixels. 


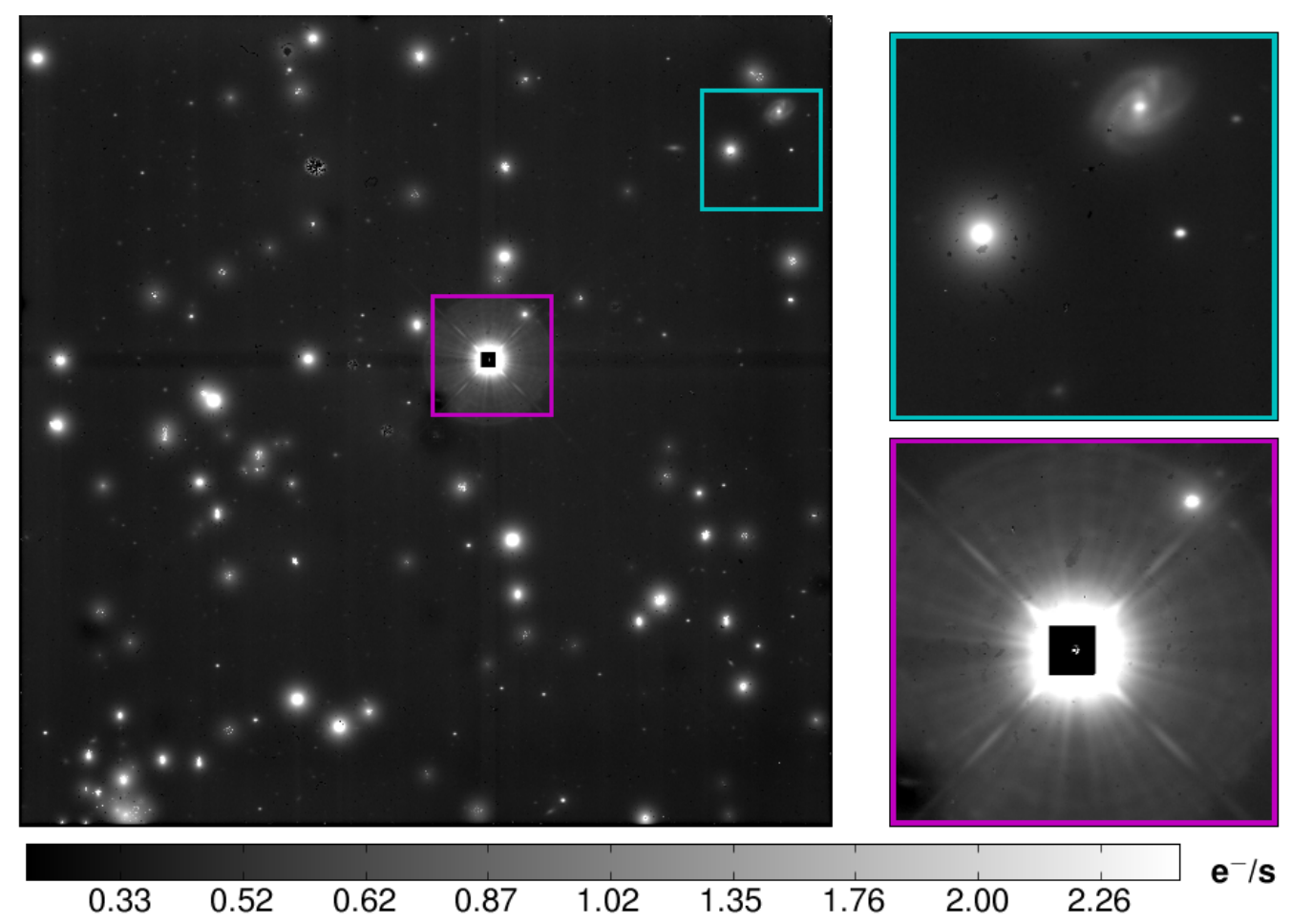

Figure 4. (Left) Full frame slopefit to a 2597 s exposure taken with H2RG-32-147 while it was operating in guide mode to guide the telescope. No flat field or dark subtraction was applied. (Right) The box blown up at the bottom right shows the guide window that has been reset constantly during the long exposure at the center of the bright star IRAS $09595+2513$. The box at upper right shows an area away from the guide window that contains two faint galaxies. Both boxes are $300 \times 300$ pixels.

Limiting Magnitude In terms of faint sources, in the guide mode slopefit we are able to detect galaxies and point sources catalogued by the Sloan Digital Sky Survey down to a magnitude of 23.1 in the i-band at the 3 $\sigma$ level. This is much fainter than the limiting magnitude in i-band of approximately 21.5 that we obtained by matching sources in the USNOA-2.0 catalog with those in our exposure with no guiding.

There is, however, a discrepancy in exposure times. To properly account for this difference, we can extrapolate for the limiting magnitude with no guiding based on theory using a standard equation for signal to noise prediction. The background sky fluxes $B$ and dark current $D$ in the exposures are very similar; the real difference is the radius subtended by the faint stars in the exposures, $r_{\text {source }}$. Figure 6 shows the expected limiting magnitude at a certain exposure time for the guiding situation where $r_{\text {source }} \sim 7.2$ pixels and $B \sim 6.8 e^{-} / s$ as well as the non-guiding situation where $r_{\text {source }} \sim 17$ pixels and $B \sim 10.6 e^{-} / s$.

The behavior is easy to understand intuitively. Essentially, when the telescope is not tracking a star properly, its light gets distributed into a large area of pixels rather than being concentrated in a small region. The light from the star then tends to blend with the background sky light and is confused with dark current, especially near the edge of the star. Taking into account the disparity in $r_{\text {source }}$, we see in Figure 6 that for an hour long exposure the guide mode will go about 1.4 magnitudes fainter.

\subsection{Saturated Pixels}

When we implemented guide mode with H2RG-32-147, resetting the pixels in the guide window did not prevent the surrounding pixels from reaching saturation. However, the reason that the surrounding pixels saturated was not because the accumulated charge from the bright star bloomed into its neighbors. Rather, the diffraction of light from IRAS $09595+2513$ into the pixels immediately surrounding the guide window is significant enough to 
generate $\sim 50 e^{-} / s$ and fill the pixel wells after about 1500 seconds. Further out, the rate of carrier generation is smaller, but still enough to saturate the pixels in 2500 seconds.

We note that the operation of guide mode will indeed prevent charge blooming if the guide window is large enough to contain all of the pixels integrating photocharge from the star. We have also used it successfully in resetting a hot pixel so that it does not spill into its neighbors. One additional benefit is that resetting the brightest portion of the stellar image prevents crosstalk between detector outputs. For $N_{\text {out }}$ detector outputs and $N_{\text {pix/row }}$ pixels per row, this effect manifests itself as $N_{\text {out }}$ repeated images of the star evenly spaced across the columns at intervals of $N_{\text {pix } / \text { row }} / N_{\text {out }}$ pixels. More details can be found in Finger et al. ${ }^{8}$

\subsection{Summary of Results}

The results from the long exposures with and without the implementation of guide mode are shown in Table 2 . As is expected for a guided camera system, the ellipticity and FWHM are reduced and the limiting magnitude is boosted when we use H2RG-32-147 to guide the Kitt Peak 2.1m telescope.
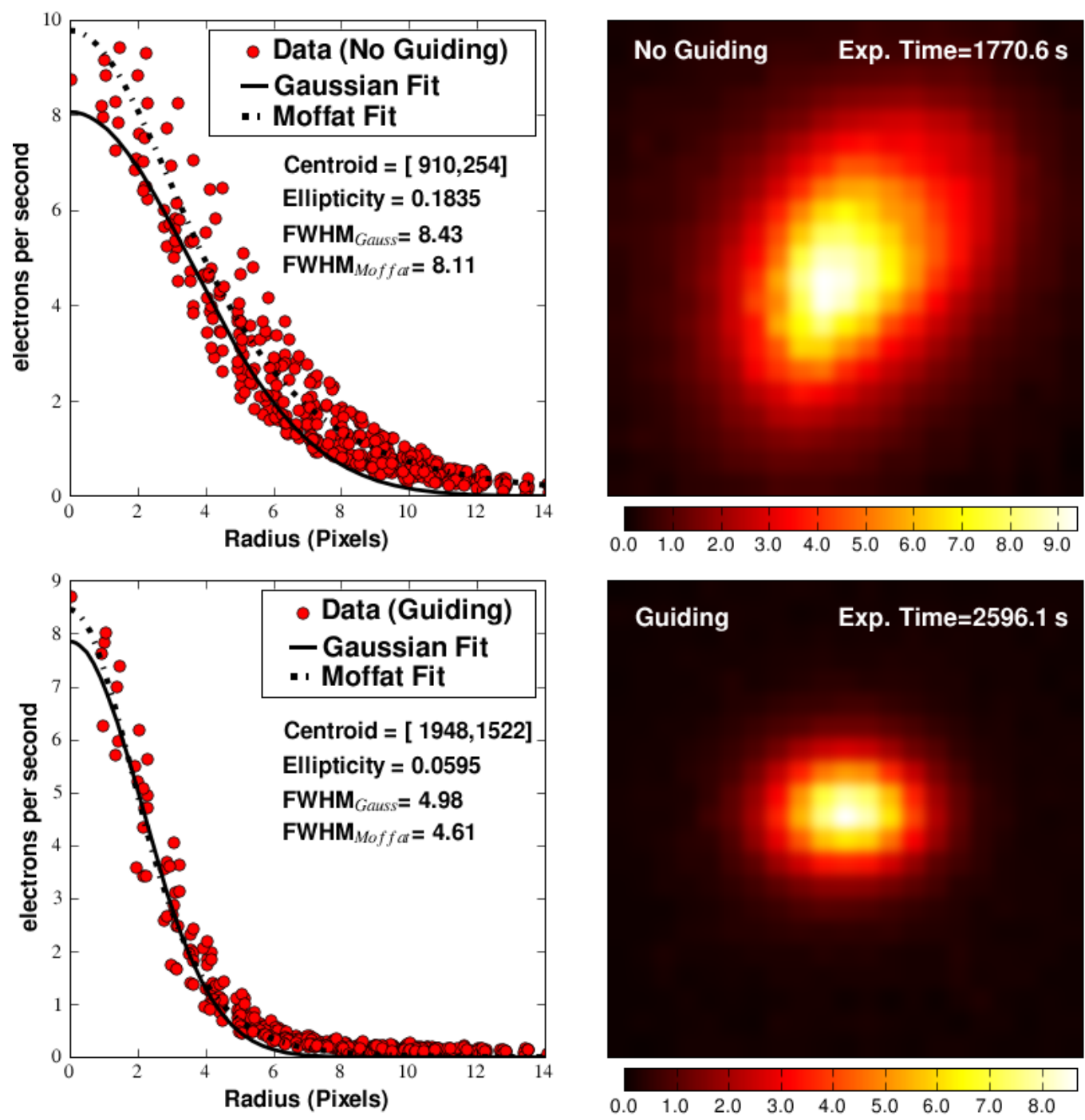

Figure 5. The top two figures show a stellar profile from a 1770 s exposure taken with H1RG-022 without telescope guiding. The bottom two show the radial profiles from a $2597 \mathrm{~s}$ exposure taken with H2RG-32-147 while it was being used in guide mode to provide offsets to the telescope. 


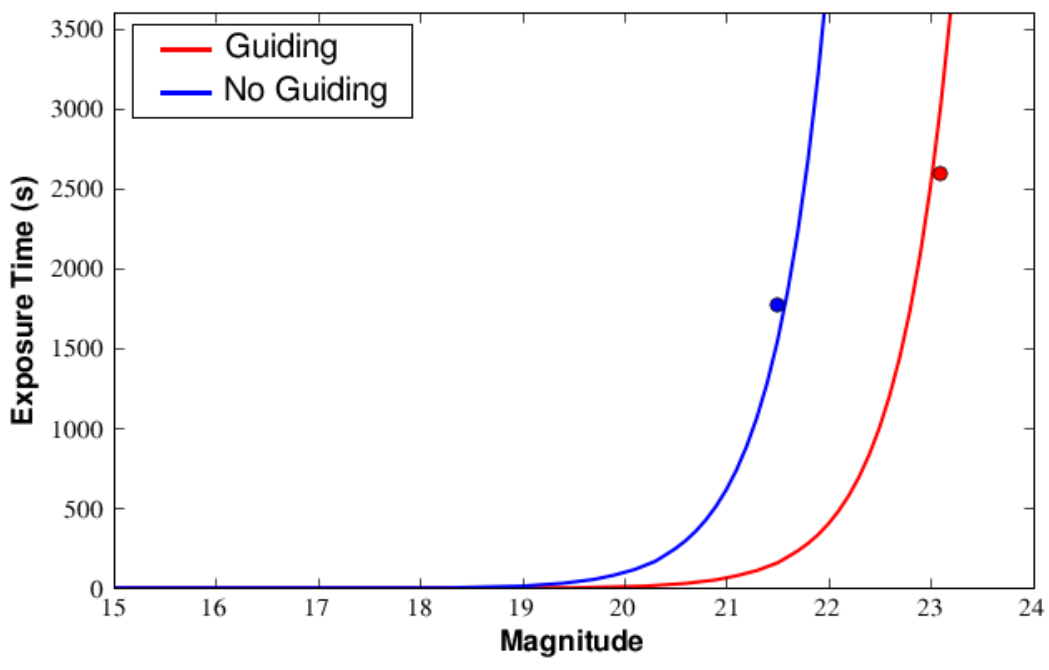

Figure 6. Theoretical exposure time required to reach limiting magnitude with a signal to noise ratio of 3 for the cases where the telescope is being guided by H2RG-32-147 (right curve) and where it is simply trying to track stars at the sidereal rate with H1RG022 (left curve). The data points are also plotted as circles. The difference is due almost entirely to the disparity in point spread functions.

\section{DISCUSSION}

\subsection{Experimental Outcome vs. Expectation}

The ellipticity and FWHM of the stars in both guided and unguided mode agree very well with the values predicted using Equation 2 along with the nightly seeing conditions and average drift rates for $m_{D E C}$ and $m_{R A}$. The measurements indicate that image persistence brought about by high flux/low fluence integrations of the guide star in window mode and pixel nonlinearity do not produce significant errors in the centroid accuracy. Based upon the brightest star used to guide, SAO 81129 (J 3.98) observed in $y$ band, this is true for fluxes < $2 \mathrm{~W} / \mathrm{m}^{2}$ and fluences $<80 \mathrm{~mJ} / \mathrm{m}^{2}$.

For future star tracking applications, it should be sufficient to consider the standard parameters of read noise, dark current shot noise, inter pixel capacitance, etc. when predicting performance, at least for fluxes and fluences below these limits. It is expected that brighter stars and longer integration times can still be used, but to address the situation concretely, additional measurements need to be carried out. Such measurements will be included in the preliminary LSST guider tests underway at RIDL and SLAC. Also, effort is being spent on modeling the latent images with finite difference simulations. If it is fruitful, the simulations might be used to estimate centroid accuracy for various flux/fluence scenarios in the future.

\subsection{Further Improvements Made in Laboratory Studies}

The results presented for the astronomical measurements were obtained with sub-optimal operating conditions for the SIDECAR ASIC and the H2RG detector. Significant improvement has been made in terms of noise and cross-talk reduction in recent laboratory measurements. The improvements are largely a result of $\mathbf{1}$ ) improved

Table 2. Quantitative comparison of long exposures obtained when the telescope was being guided by H2RG-32-147 and simply tracking at the sidereal rate

\begin{tabular}{lcc}
\hline Parameter & No Guiding with H1RG-022 & Guide Mode with H2RG-32-147 \\
\hline \hline Ellipticity $(\|\vec{e}\|)$ & 0.18 & 0.06 \\
FWHM $_{\text {Gauss }}($ pixels $) /\left({ }^{\prime \prime}\right)$ & $8.70(2.02)$ & $5.90(1.37)$ \\
FWHM $_{\text {Moffat }}($ pixels $) /\left({ }^{\prime \prime}\right)$ & $7.24(1.67)$ & $4.60(1.07)$ \\
Atmospheric Seeing $($ pixels $) /\left({ }^{\prime \prime}\right)$ & $3.9(0.90)$ & $4.31(1.00)$ \\
$M_{\text {Lim }}$ in 3600s exposure & 21.9 & 23.3 \\
\hline
\end{tabular}



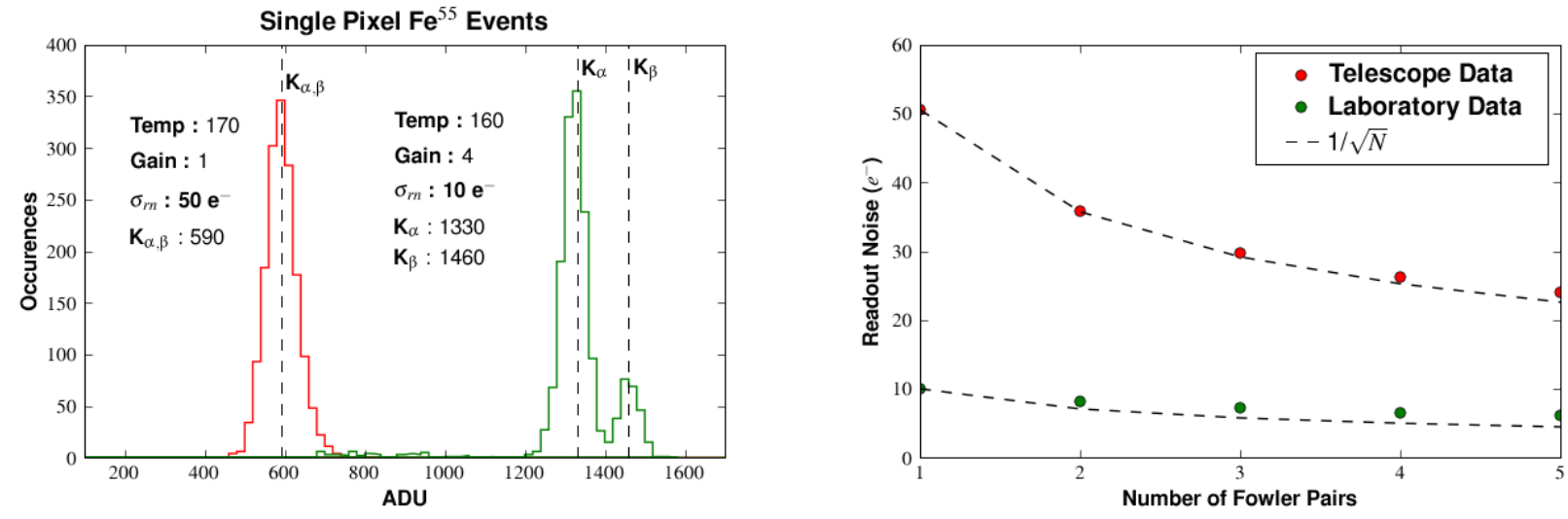

Figure 7. Left) Comparison of Fe55 histograms from data taken while at telescope (left histogram) and in laboratory after noise reduction (right histogram). The separate peaks are clearly visible in the laboratory data, indicating noise improvement and diminished crosstalk. Right) Comparison of read noise from data taken while at telescope (top curve) and laboratory data (bottom curve). The temperatures and gains for the two curves are the same as the ones in the left plot.

detector and SIDECAR bias voltages and currents, 2) use of a cryogenic SIDECAR as opposed to the warm development board ${ }^{\ddagger}, \mathbf{3}$ ) use of a linear power supply to power the SIDECAR instead of USB power, 4) averaging single detector outputs on multiple SIDECAR channels to lower the noise contribution of the ADCs, 5) increased preamp gain on the SIDECAR to lower the noise contribution of the ADCs, 6) increasing the substrate voltage $V_{S U B}$ from 15 to 40 volts, 7) use of unbuffered mode instead of buffered mode, and 8) use of the reference output $V_{R E F O U T}$ on the H2RG detector instead of an internal SIDECAR reference for differential measurement of the detector video outputs.

For a detailed discussion of points 1-3, the reader is referred to Dorn et al. ${ }^{6}$ and for $\mathbf{4 - 5}$, to Loose et al. ${ }^{11}$ With regard to point $\mathbf{5}$, it should also be mentioned that a pre-amp gain of 4 on the SIDECAR was determined to be optimum as it is the highest gain-higher gain corresponds to lower input referred noise since the noise in ADU is roughly constant - which still allows one to realize the full dynamic range of the detector pixels. This gain corresponds to roughly $16 \mu V / A D U$, and with the inverse nodal capacitance of about $11 \mu V / e^{-}$and a well depth of 100,000 $e^{-}$, the full well range of a typical pixel corresponds to 68,000 ADU, just above the 16 bit maximum. For point $\mathbf{6}$, the improvements gained upon increasing $V_{S U B}$ follow from a decrease in lateral charge diffusion and read noise. Point $\mathbf{7}$ addresses the noise associated with the output source follower FET. The output source follower might be necessary to drive a large load capacitance, as was the case for our telescope observations in which the analog detector output signals had to travel from the detector inside the dewar to the SIDECAR outside the dewar over about 30" of cable. However, the output FET requires a pullup resistor or current source, both of which introduce current shot noise and nonlinearity. The use of $V_{R E F O U T}$ mentioned in point $\mathbf{8}$ was perhaps the most significant noise reduction measure and is discussed below.

Figure 7 illustrates the improvement in performance brought about by the adjustments described. Most notable is the reduction in CDS read noise from $\sigma_{r n}=50 e^{-}$to $\sigma_{r n}=10 e^{-}$rms. The very large read noise present in the telescope data is mainly due to a high frequency noise component present on the $V_{R E F M A I N}$ SIDECAR reference voltage and improper internal SIDECAR bias voltages. This component was eliminated in the laboratory data by using the $V_{R E F O U T}$ signal of the H2RG instead of $V_{R E F M A I N}$ for differential measurement. Measurements of $\mathrm{Fe}^{55}$ spectra made at the telescope reflect the high read noise in a failure to discern the $K_{\alpha}$ and $K_{\beta}$ peaks. The contribution of $\sigma_{r n}$ to the width of the peaks is significantly greater than that of $\sigma_{\text {fano }}$ and

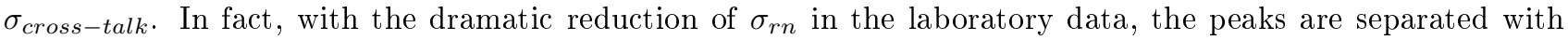
a substrate voltage of only $V_{S U B}=10$ Volts, indicating that the cross talk contribution is negligible. It should

\footnotetext{
${ }^{\ddagger}$ In addition to the ASIC chip being cooled to cryogenic temperatures, the length of wire over which the analog video signals had to travel was decreased from about 30 " to 2 ".
} 
also be noted that the CDS read noise drops significantly with a decrease in temperature. At $100 \mathrm{~K}$, it drops to approximately $8 e^{-}$from $10 e^{-}$at $170 \mathrm{~K}$.

\section{CONCLUSIONS}

A HyViSI H2RG has been used successfully to guide the Kitt Peak $2.1 \mathrm{~m}$ telescope while simultaneously obtaining near seeing and background-limited astronomical images in guide mode. Despite concerns of pixel non-linearity, crosstalk, and charge persistence, the guide window successfully reports an accurate centroid. Further improvements are expected for low brightness guide stars based upon a decrease in read noise and $\mathrm{Fe}^{55}$ spectra obtained in laboratory data accumulated after the telescope observations.

\section{ACKNOWLEDGEMENTS}

The tests described were made possible by the DOE, NSF, Rochester Institute of Technology Imaging Detector Laboratory (RIDL), and Teledyne Scientific and Imaging (TSI). Initial code development was performed at RIDL with support from a NYSTAR Faculty Development Program grant. We thank Markus Loose, Raphael Ricardo, Mark Farris, Yibin Bai, Jim Beletic, and the other members of TSI for all of their help in improving detector performance. Research was done in part as Visiting Astronomers, Kitt Peak National Observatory, National Optical Astronomy Observatory, which is operated by the Association of Universities for Research in Astronomy (AURA) under cooperative agreement with the National Science Foundation. We thank Dick Joyce, Skip Andre, and the KPNO staff for all of their excellent help with our telescope observations. This research has made use of the SIMBAD database, operated at CDS, Strasbourg, France.

\section{REFERENCES}

1. Y. Bai, S. G. Bernd, J. R. Hosack, M. C. Farriss, J. T. Montroy, and J. Bajaj, "Hybrid CMOS focal plane array with extended UV and NIR response for space applications," SPIE Proc. 48, 2003.

2. Y. Bai, J. Bajaj, J. W. Beletic, M. C. Farris, A. Joshi, S. Lauxtermann, A. Petersen, and G. Williams, "Teledyne imaging sensors: Silicon CMOS imaging technologies for x-ray, uv, visible and near infrared," SPIE Proc. 7021(01), 2008.

3. L. M. Simms, D. F. Figer, B. J. Hanold, D. J. Kerr, D. K. Gilmore, S. M. Kahn, and J. A. Tyson, "First use of a HyViSI H4RG for astronomical observations," SPIE Proc 6690, August 2007.

4. B. N. Dorland, G. S. Hennessy, N. Zacharias, D. G. Monet, H. Harris, C. Rollins, P. Shu, L. Miko, B. Mott, A. Waczynski, and E. Kan, "Laboratory and sky testing results for the TIS H4RG-10 4kx4k, 10 micron visible CMOS-hybrid detector," SPIE Proc. 6690, 2007.

5. B. N. Dorland, R. Dudik, G. S. Hennessy, A. Waczynski, B. J. Rauscher, P. K. Shu, and J. E. Hubbs, "Laboratory and sky testing of the second generation Teledyne Imaging Sensor (TIS) 16.7 megapixel visible/near-IR CMOS-hybrid array H4RG-10 A2," SPIE Proc 7439, 2009.

6. R. J. Dorn, S. Eschbaumer, D. N. B. Hall, G. Finger, L. Mehrgan, M. Meyer, and J. Stegmeier, "Evaluation of the teledyne sidecar asic at cryogenic temperature using a visible hybrid H2RG focal plane array in 32 channel readout mode," SPIE Proc 7021-29, 2008.

7. A. Hoffman, M. Loose, and V. Suntharalingam, "CMOS detector technology" in Scientific Detectors For Astronomy 2005, pp. 377-402, Springer Netherlands, 2006.

8. G. Finger, R. J. Dorn, S. Eschbaumer, D. N. B. Hall, L. Mehrgan, M. Meyer, and J. Stegmeier, "Performance evaluation, readout modees, and calibration techniques of HgCdTe HAWAII-2RG mosaic arrays," SPIE Proc 7021-29, 2008.

9. D. Bacon, A. Refregier, and R. Ellis, "Detection of weak gravitational lensing by large-scale structure," Mon. Not. Roy. Astron. Soc. 318, p. 625, 2000.

10. J. Koornneef, "Near-infrared photometry: Ii. intrinsic colours and the absolute calibration from one to five microns," Astronomy and Astrophysics 128, pp. 84-93, 1983.

11. M. Loose, J. Beletic, J. Garnett, and M. Xiu, "High-performance focal plane arrays based on the HAWAII2RG/4RG and the SIDECAR ASIC," SPIE Proc 6690, 2007. 